\title{
Interacciones entre el mejillón dorado y macroinvertebrados bentónicos nativos del Río Uruguay
}

\author{
Interactions between the golden mussel and native benthic \\ macroinvertebrates of Uruguay River
}

\section{Interações entre o mexilhão dourado e os macroinvertebrados bentônicos nativos do Rio Uruguai}

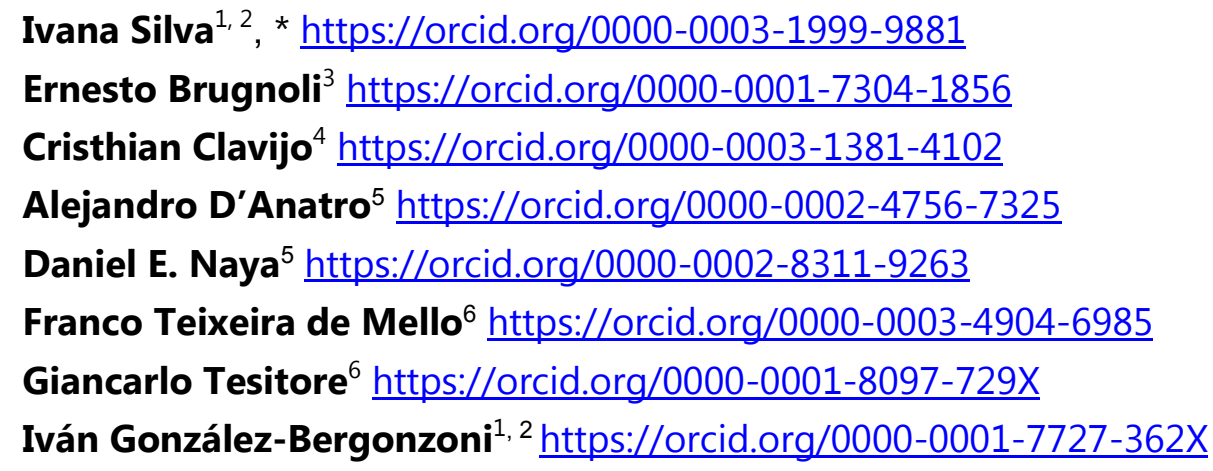

*Autor de contacto: isilva@fcien.edu.uy

${ }^{1}$ Departamento del Agua, CENUR Litoral Norte, Universidad de la República, Paysandú, Uruguay

${ }^{2}$ Departamento de Ecología y Biología Evolutiva, Instituto de Investigaciones Biológicas Clemente Estable, Montevideo, Uruguay

${ }^{3}$ Instituto de Ecología y Ciencias Ambientales - Oceanografía y Ecología

Marina, Facultad de Ciencias, Universidad de la República, Montevideo, Uruguay

${ }^{4}$ Museo Nacional de Historia Natural, Montevideo, Uruguay

${ }^{5}$ Departamento de Ecología y Evolución, Facultad de Ciencias, Universidad de la República, Montevideo, Uruguay

${ }^{6}$ Departamento de Ecología y Gestión Ambiental CURE, Universidad de la República, Maldonado, Uruguay

Recepción: 7 Setiembre 2020 Aprobación: 12 Mayo 2021

Esta obra está bajo una Licencia Creative Commons Atribución-NoComercial 4.0 Internacional. 
Resumen: Los efectos que Limnoperna fortunei ocasiona sobre los ecosistemas vienen siendo estudiados en los últimos años a nivel nacional. No obstante, las interacciones que este invasor establece con la fauna bentónica nativa no han sido determinadas en detalle. El objetivo de este estudio fue recopilar información sobre las interacciones entre el mejillón dorado y macroinvertebrados nativos del Río Uruguay, a través de (a) relevamiento bibliográfico, (b) registro fotográfico obtenido en campo y (c) análisis de datos de un experimento de colonización realizado previamente en litorales rocosos de este río. Las interacciones reportadas en la bibliografía refirieron mayoritariamente a interacciones directas de biofouling de L. fortunei sobre especies de bivalvos y gasterópodos nativos, con potencial efecto negativo para estos individuos. La documentación fotográfica permitió reportar, por primera vez en la región, una interacción directa de $L$. fortunei con la familia Spongillidae, mediante la cual estos poríferos cubren y sofocan a mejillones ya asentados. Finalmente, a través del experimento realizado in situ se encontró que el mejillón genera efectos negativos indirectos sobre algunos macroinvertebrados bentónicos nativos. Según el análisis de datos, una alta abundancia de mejillones asentados se relaciona con una reducción en las abundancias de dos de los grupos bentónicos nativos dominantes de este río, como son los gasterópodos y dípteros.

Pallabras clave: Limnoperna fortunei, biofouling, fauna bentónica nativa, invasiones biológicas.

Abstract: The ecosystem effects of Limnoperna fortunei have been studied nationally in recent years. However, the interactions that this invader establishes with the native benthic fauna have not been determined in detail. The objective of this study was to collect information on the interactions between the golden mussel and macroinvertebrates native to the Uruguay River, through (a) bibliographic survey, (b) photographic record obtained in the field and (c) analysis of data from an experiment of colonization carried out previously on rocky shores of this river. The interactions reported in the bibliography mainly referred to direct interactions of $L$. fortunei biofouling on native bivalve and gastropod species, with a potential negative effect for these individuals. The photographic documentation specifically made it possible to report for the first time in the region, a direct interaction of $L$. fortunei with the Spongillidae family, through which these porifers cover and suffocate already settled mussels. Finally, through the experiment carried out in situ, it was found that the mussel generates indirect negative effects on some native benthic macroinvertebrates. According to the data analysis, a high abundance of settled mussels is related to a reduction in the abundances of two of the dominant native benthic groups of this river, such as gastropods and dipterans.

Keywords: Limnoperna fortunei, biofouling, native benthic fauna, biological invasions.

Resumo: Os efeitos que o Limnoperna fortunei causa nos ecossistemas foram estudados nos últimos anos em nível nacional. No entanto, as interações que este invasor estabelece com a fauna bentônica nativa não foram determinadas em detalhes. $O$ objetivo deste estudo foi coletar informações sobre as interações entre o mexilhão dourado e macroinvertebrados nativos do Rio Uruguai, por meio de (a) levantamento 
bibliográfico, (b) registro fotográfico obtido em campo e (c) análise de dados de um experimento de colonização realizada anteriormente em costões rochosos deste rio. As interações relatadas na bibliografia referem-se principalmente às interações diretas de $L$. fortunei bioincrustantes em espécies nativas de bivalves e gastrópodes, com potencial efeito negativo para esses indivíduos. A documentação fotográfica especificamente possibilitou relatar, pela primeira vez na região, uma interação direta de $L$. fortunei com a família Spongillidae, através da qual esses poríferos cobrem e sufocam mexilhões já assentados. Por fim, por meio do experimento realizado in situ, constatou-se que o mexilhão gera efeitos negativos indiretos sobre alguns macroinvertebrados bentônicos nativos. De acordo com a análise dos dados, uma alta abundância de mexilhões assentados está relacionada a uma redução nas abundâncias de dois grupos bentônicos nativos dominantes deste rio, como gastrópodes e dípteros.

Palavras-chave: Limnoperna fortunei, bioincrustação, fauna bentônica nativa, invasões biológicas.

\section{INTRODUCCIÓN}

El mejillón dorado (Limnoperna fortunei) es una de las especies invasoras de ecosistemas de agua dulce de Sudamérica que ha provocado mayor interés para la región debido a los altos costos económicos que genera (Cataldo, et al., 2003; Brugnoli, et al., 2006; Boltovskoy, et al., 2015). Desde su ingreso al Río de la Plata en la década del 90 (Pastorino, et al., 1993; Scarabino y Verde, 1995), comenzó una rápida dispersión, encontrándose actualmente en varios afluentes del Río Uruguay, Río Negro, Río Santa Lucía, Río de la Plata, Laguna Merín y Laguna del Sauce (Brugnoli, et al., 2005; Brugnoli, et al., 2011; Clemente, et al., 2015). El éxito en la dispersión y asentamiento del mejillón dorado está dado por las características biológicas de la especie. Estos bivalvos, de hábito epifaunal, son organismos dioicos de fertilización externa que producen un importante número de larvas de vida libre (Ezcurra de Drago, et al., 2006). Estas larvas presentan varios estadios de desarrollo, y el último de ellos, la larva plantígrada (Ezcurra de Drago, et al., 2006), puede fijarse en distintos tipos de sustratos duros (natural o artificial) por medio de un fuerte biso (Darrigran, 2002). Así, la especie puede asentarse fácilmente en un nuevo sitio, formando agregaciones de miles de individuos por metro cuadrado en un corto periodo de tiempo (Darrigran, 2002).

Estas agregaciones pueden generar importantes incrustaciones en infraestructuras de industrias y centrales hidroeléctricas que utilizan el agua de río, obstruyendo diferentes tipos de filtros y causando daños en las plantas de purificación de agua (Cataldo, et al., 2003; Brugnoli, et al., 2006; Boltovskoy, et al., 2015). Además, esta invasión provoca importantes daños en embarcaciones, ya que ejemplares de $L$. fortunei pueden adherirse a sus cascos, así como a otras partes sumergidas o en contacto con el agua (filtros, hélices, válvulas), generando problemas para su funcionamiento e hidrodinámica (Pérez, 2012; Boltovskoy, et al., 2015). Este fenómeno, conocido como macrofouling, implica grandes pérdidas 
económicas para el país debido al aumento de las tareas adicionales de mantenimiento de las estructuras afectadas y la generación de distintos planes de manejo de la especie (Brugnoli, et al., 2006).

Por otra parte, se ha demostrado que la invasión del mejilón dorado implica diversas consecuencias ecológicas. En este sentido, esta especie puede actuar como un agente estructural dominante (Crooks, 2002), generando alteraciones sobre distintos niveles del ecosistema, modificando la estructura trófica, el hábitat físico, la disponibilidad y/o calidad de nutrientes, entre otras (por ejemplo, Boltovskoy, et al., 2009; Sylvester, et al., 2007; Duchini, et al., 2018). Específicamente, debido a la actividad filtradora de L. fortunei (Sylvester, et al., 2005; Cataldo, et al., 2012a), la misma puede producir una disminución del plancton y un consecuente aumento de la disponibilidad de nutrientes (Cataldo, et al., 2012a). En línea con esto, una disminución del material en suspensión puede implicar, a su vez, un aumento en la transparencia del agua (Boltovskoy, et al., 2009). Además, el material particulado extraído de la columna de agua es posteriormente transferido al sustrato bentónico en forma de heces y pseudoheces, generando un enriquecimiento en los sedimentos del río (Welker y Walz, 1998). La modificación del sustrato podría generar finalmente una mayor disponibilidad de alimento para algunos taxa y espacios disponibles como refugio ante depredadores (Darrigran, et al.,1998; Darrigran, 2002; Sylvester, et al., 2007; Sardiña, et al., 2008).

En investigaciones previas realizadas en los principales sistemas hídricos invadidos de la cuenca del Plata, como el Río Paraná y el Río de la Plata, se ha demostrado que los cambios producidos por $L$. fortunei en el ambiente implican, a su vez, importantes efectos sobre la fauna nativa, particularmente sobre los macroinvertebrados bentónicos (Mansur, et al., 1999; 2003; Sylvester, et al., 2007; Boltovskoy y Correa, 2015; Linares, et al., 2017; Duchini, et al., 2018). La mayoría de los estudios describen a este organismo invasor como un facilitador de varios grupos de macroinvertebrados bentónicos (por ejemplo, Oligochaeta, Nematoda, Hirudinea), viéndose posiblemente beneficiados por una mayor disponibilidad de recursos alimenticios y refugio ante depredadores, aumentando su abundancia en sitios colonizados (Sylvester, et al., 2007; Boltovskoy y Correa, 2015; Duchini, et al., 2018). Por otra parte, la literatura ha sugerido que probablemente esta invasión genera importantes impactos negativos sobre otros grupos de invertebrados nativos. Linares y otros (2017) destacan la presencia conjunta de $L$. fortunei con otro bivalvo invasor, como Corbicula fluminea, en varios embalses colonizados en Brasil, donde pueden disminuir las abundancias de algunos grupos, tales como Chironomidae, debido a probables cambios en los patrones de sedimentación del fondo del río. En tanto, Mansur y otros (1999 y 2003) reportaron el asentamiento de $L$. fortunei sobre gasterópodos, como Pomacea canaliculata, y bivalvos nativos, como Diplodon koseretzi y Leila blainvilliana, provocando una posible disminución de estos moluscos por oclusión, 
impedimento para abrir y cerrar sus valvas; lo que directamente afecta su capacidad de ingestión y, por lo tanto, los animales mueren. Asimismo, otros autores han informado que en el Río Paraná este mejillón tiene la capacidad de fijarse a cangrejos nativos, como Aegla platensis o Trichodactylus borellianus, adhiriéndose incluso a individuos de menor peso y longitud que los del mismo mejillón (Lopes, et al., 2009; Rojas Molina y Williner, 2013).

Para Uruguay, y específicamente para grandes ríos de nuestro país, las interacciones entre Limnoperna e invertebrados nativos no han sido identificadas en detalle. Sin embargo, en los últimos años, se ha avanzado en la comprensión de la ecología de esta invasión, y de sus principales efectos sobre el ecosistema, específicamente en el Río Uruguay (Cataldo, et al., 2012b; Eugui, et al., 2012; Silva, et al., 2020). El objetivo del presente trabajo es recopilar la información disponible sobre las interacciones entre $L$. fortunei y macroinvertebrados bentónicos nativos del Río Uruguay, obtenida en primera instancia de investigaciones publicadas, de registros fotográficos puntuales realizados en campo, y de un experimento de colonización de mejillones y fauna acompañante in situ, realizado en litorales rocosos del bajo Río Uruguay.

\section{MATERIALES Y MÉTODOS}

\section{Revisión bibliográfica}

Se realizó una búsqueda bibliográfica en el buscador "Google Scholar", utilizando las palabras clave: "Río Uruguay, Uruguay River, Limnoperna fortunei, mejillón dorado, golden mussel, invertebrados bentónicos, benthic invertebrates", seleccionando aquellos trabajos que documentaron interacciones entre esta especie invasora y diferentes grupos de macroinvertebrados bentónicos de dicho río.

\section{Registro fotográfico}

Se solicitó a los grupos de investigación que integran los autores del presente trabajo registros fotográficos de interacciones entre mejillón e invertebrados observadas en campo y en diferentes sitios del Río Uruguay. Estos registros se basaron principalmente en el biofouling sobre otros organismos y la potencial competencia por espacio. 
Experimento de colonización de mejillones y fauna bentónica acompañante in situ

Mediante un experimento in situ en litorales rocosos del Río Uruguay, se analizó la relación entre abundancias de $L$. fortunei y de macroinvertebrados nativos dominantes.

Se construyeron ocho jaulas ( $1.0 \mathrm{~m}$ de largo $\times 1.0 \mathrm{~m}$ de ancho $\times 0.5 \mathrm{~m}$ de alto) cubiertas con una malla metálica (tamaño de malla: $5.0 \mathrm{~mm}$ ). En el fondo de cada una de estas jaulas se colocaron 32 ladrillos, cubriendo todo el piso, los cuales funcionaron como sustratos artificiales para el asentamiento de los organismos. Como fue demostrado en trabajos previos (de Melo Rosa, et al., 2019), los peces pueden ejercer un importante control sobre la abundancia del mejilón; por lo tanto, las jaulas fueron diseñadas de forma estratégica: cuatro de ellas cubiertas completamente por tejido (evitando el acceso de depredadores) y otras cuatro con dos de sus lados laterales desprovistos de tejido (permitiendo el acceso a depredadores). Este diseño aseguró la existencia de un importante gradiente en las abundancias del mejillón, permitiendo analizar las correlaciones entre su abundancia y la de los macroinvertebrados nativos.

El área de estudio se localizó en el balneario Las Cañas (34 10'25 "S, 58²1'29" O), departamento de Río Negro (Figura 1a, b). En setiembre de 2016 se inició el experimento a efectos de anteponerse al periodo de asentamiento larval, el cual ocurre, según varios autores, en los meses de primavera, cuando la temperatura del agua supera los $17^{\circ} \mathrm{C}$ (Darrigran, et al., 2002; Brugnoli et al., 2011).

Las jaulas fueron ancladas firmemente al fondo del río, a una distancia de 2 a 10 m entre ellas; y se intercalaron según el tipo de tratamiento: acceso y exclusión de depredadores. Las profundidades a las que se colocaron las jaulas variaron entre 1.2 y $1.7 \mathrm{~m}$ en promedio durante el período de muestreo, debido a las fluctuaciones del nivel del río; sin embargo, en ningún caso la profundidad entre tratamientos fue diferente (ANOVA, $g l=16, F=0.062 ; p=0.8$ ). Asimismo, cuatro jaulas (dos de acceso y dos de exclusión) fueron colocadas en una bahía (con poca influencia de las olas), y otras cuatro en una zona expuesta a mayor oleaje (a aproximadamente $20 \mathrm{~m}$ de la bahía), para integrar los potenciales factores de variación y representar las condiciones ambientales del sitio.

El experimento se llevó a cabo a lo largo de 181 días, y se tomaron muestras cada 15-20 días. Finalmente, los módulos fueron retirados del río en marzo de 2017. Entre los muestreos del día 110 y el día 181, la inspección visual de los sustratos indicó claramente que el asentamiento de mejillones había ocurrido, registrándose pocos ejemplares para el día 110 (268 ind. $\mathrm{m}^{-2}$ en promedio, considerando ambos tratamientos) en comparación con el día 181 (763 ind. $\mathrm{m}^{-2}$ en promedio, considerando ambos tratamientos) (ANOVA, $d f=45, F=70.44$, 
$p<0.0001)$. Por tanto, con el fin de estudiar la relación entre abundancias de mejillón y los macroinvertebrados nativos encontrados, se tomó al muestreo del día 110 como el periodo de tiempo en el que el mejillón estuvo ausente (o en bajas densidades) y al muestreo del día 181 como el periodo posterior a la colonización de los sustratos, es decir, cuando el mejillón estuvo presente (y en altas densidades).

En cada muestreo se seleccionaron al azar tres ladrillos de cada módulo y se procedió a remover todos los mejillones y macroinvertebrados nativos allí asentados, utilizando diferentes pinzas y filtrando cada muestra por un tamiz de $300 \mu \mathrm{m}$ de apertura de malla. Las muestras obtenidas se conservaron en alcohol 95\% para su posterior análisis. En el laboratorio de Ecología y Biología Evolutiva del Instituto de Investigaciones Biológicas Clemente Estable (IIBCE), los macroinvertebrados recolectados fueron colocados en una placa de Petri y con la ayuda de lupa binocular y claves taxonómicas (Costa, et al., 2006; Domínguez, et al., 2006; Domínguez y Fernández, 2009), cada ejemplar fue identificado al menor nivel taxonómico posible.

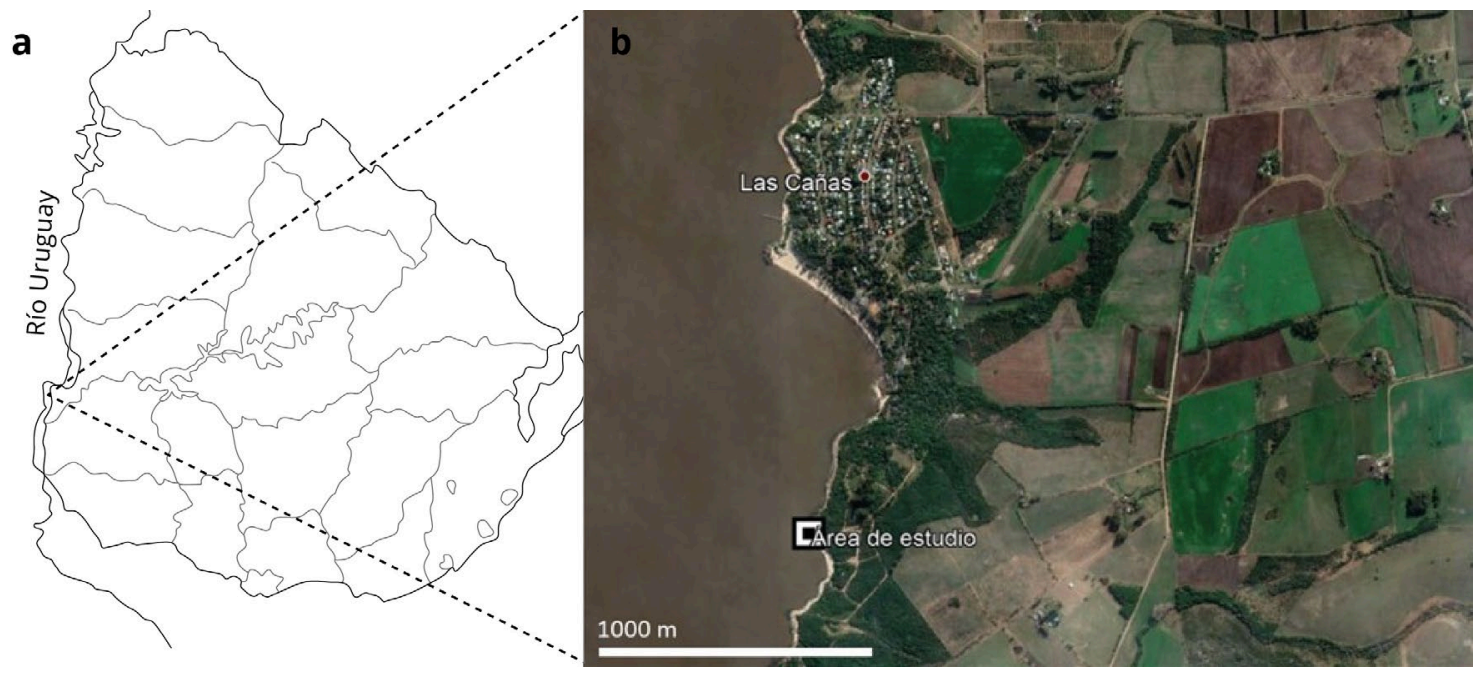

Figura 1. a) Mapa de Uruguay indicando la ubicación del balneario Las Cañas, en la porción inferior del Río Uruguay. b) Imagen de Google Earth mostrando el área de estudio localizada en Las Cañas, Río Negro.

Los ejemplares identificados fueron clasificados según la abundancia de los grupos más frecuentes (aquellos presentes en al menos el $60 \%$ de los sustratos).

La relación entre la abundancia de mejillones y de cada grupo de macroinvertebrados (teniendo en cuenta ambos muestreos, día 110 y 181 del experimento) fue analizada mediante el ajuste de modelos lineales generalizados mixtos (GLMM, por sus siglas en inglés, $\alpha=0.05)$, considerando la abundancia de L. fortunei como variable explicativa categórica de efecto fijo, y la jaula como 
variable de efecto aleatorio. Dado que los datos de abundancia de cada grupo de invertebrados fueron tomados como conteos, se probaron dos distribuciones diferentes (gamma y poisson) con el fin de buscar aquella que mejor se ajustara a dicha variable de respuesta. La selección de la distribución de cada variable de respuesta se realizó, en todos los casos, mediante la función fitdist del paquete fitdistrplus (Delignette-Muller y Dutang, 2015), y se comprobó la bondad de ajuste de estas distribuciones con la función gofstat en el software $R$. Finalmente, la validación de cada uno de los modelos ajustados se realizó mediante los gráficos de diagnosis de los residuos (de tipo Pearson) (Logan, 2010), en $R$.

\section{RESULTADOS}

\section{Revisión bibliográfica y registro fotográfico}

De acuerdo con los criterios de búsqueda escogidos, no se encontraron publicaciones con foco en los efectos de la invasión de L. fortunei sobre la fauna bentónica nativa del Río Uruguay. Sin embargo, se encontraron algunos trabajos presentados en congresos y evidencia fotográfica que indican interacciones puntuales de este bivalvo invasor con otros organismos. Específicamente, Eugui y otros (2012) reportaron biofouling de Limnoperna sobre algunos gasterópodos, como Pomacea insularum y P. megastoma. Asimismo, en el bajo Río Uruguay se han registrado ejemplares de mejillón dorado asentados incluso dentro de algunos bivalvos nativos vivos, como Leila blainvilliana (Cristhian Clavijo, obs. pers., Figura 2a), particularmente en la localidad de Fray Bentos, Departamento de Río Negro. Además, se han registrado asentamientos puntuales de mejillones sobre grandes gasterópodos, como P. maculata, en la localidad de Nueva Palmira, Departamento de Colonia (Cristhian Clavijo, obs. pers., Figura 2b).
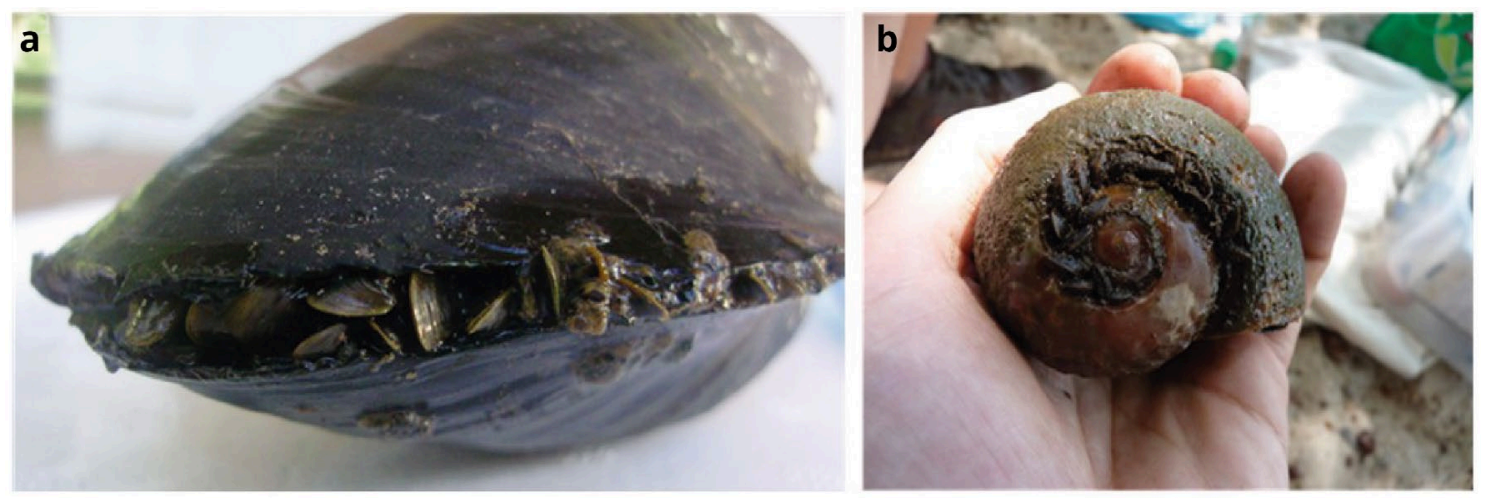

Figura 2. Ejemplares de mejillón dorado dentro de L. blainvilliana (a) y sobre $P$. maculata (b). Fotos: Cristhian Clavijo.

Por otra parte, se han registrado mejillones dorados cubiertos parcial o totalmente por ejemplares de Trochospongilla repens, en la zona de Fray Bentos 
(Cristhian Clavijo, obs. pers., Figura 3a) y sobre otras esponjas de agua dulce sin identificar, en la localidad de Bella Unión, Departamento de Artigas (Sebastián Gómez Barboza, obs. pers., Figura 3b-d), en la localidad La Concordia, Departamento de Soriano, y en San Javier (Iván González-Bergonzoni, obs. pers., Figura 3e y f, respectivamente) y Casablanca (Iván González-Bergonzoni, obs. pers. Figura 3g), Departamento de Paysandú. Esta documentación gráfica, junto con observaciones de campo, demuestra que las especies de esponjas nativas tienen la capacidad de cubrir las colonias de mejillones asentados en sustratos duros.
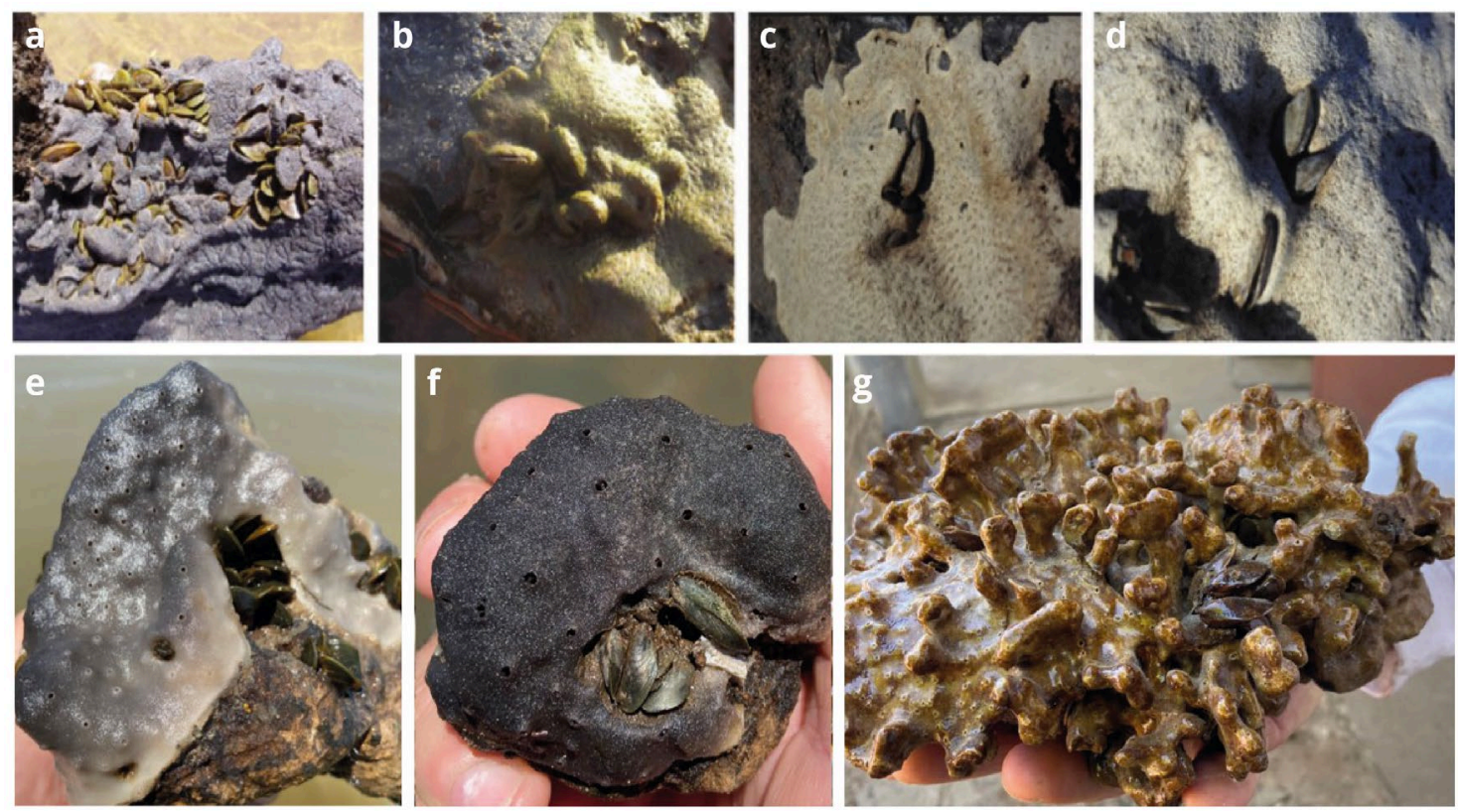

Figura 3. a) Individuos de mejillón dorado siendo cubiertos por Trochospongilla repens (Porífera, Spongillidae), Foto: Cristhian Clavijo. b-g) Individuos de mejillón dorado siendo cubiertos por ejemplares de la familia Spongillidae, especie sin identificar. Fotos: Sebastián Gómez-Barboza e Iván González-Bergonzoni.

\section{Experimento de exclusión y colonización in situ}

Se colectaron e identificaron 33 taxa de macroinvertebrados, considerando ambos muestreos (antes y después del asentamiento masivo del mejillón) (Tabla 1). Dentro de los macroinvertebrados identificados, se destacan los gasterópodos (Potamolitus spp., Heleobia spp., Chilinidae, entre otros) y los Chironomidae. Además, se lograron identificar cuatro familias de efemerópteros (Baetidae, Caenidae, Leptohyphidae, Leptophlebiidae), dos familias de tricópteros (Polycentropodidae y Philopotamidae), una familia de coleópteros (Elmidae), una familia de hemípteros (Corixidae) y dos tipos de almejas (Eupera sp. y Curbiculidae), entre otros (Tabla 1). 
Por otra parte, agrupando los taxa colectados en niveles taxonómicos superiores (Subclase y Orden), se encontró que los gasterópodos, dípteros, efemerópteros y tricópteros fueron los grupos destacados, estando presentes en el $100 \%, 98 \%, 96 \%$ y $64 \%$ de los sustratos, respectivamente. Estos cuatro grupos se catalogan como macroinvertebrados frecuentes.

Tabla 1. Densidad media ( \pm desviación estándar; ds) de todos los macroinvertebrados recolectados en cada tratamiento (acceso y exclusión) para cada muestreo: antes y después del asentamiento masivo de mejillones en los sustratos. Se encuentran ordenados por Filo (en orden alfabético), luego por Subclase u Orden, y finalmente por el mínimo nivel taxonómico identificado: Familia, Subfamilia, Tribu, Género y Especie. Se muestra la densidad total promedio y la riqueza de taxa. * $\mathrm{s} / \mathrm{i}=$ taxón sin identificar. 


\begin{tabular}{|c|c|c|c|c|c|c|}
\hline \multirow[t]{2}{*}{ Filo } & \multirow{2}{*}{$\begin{array}{l}\text { Subclase } \\
\text { /Orden }\end{array}$} & \multirow{2}{*}{$\begin{array}{c}\text { Familia } \\
\text { Subfamilia/Tribu/ } \\
\text { Género/Especie }\end{array}$} & \multicolumn{2}{|c|}{ Antes } & \multicolumn{2}{|c|}{ Después } \\
\hline & & & Acceso & Exclusión & Acceso & Exclusión \\
\hline \multirow[t]{2}{*}{ Annelida } & Hirudinea & Hirudinea s/i & $1.0 \pm 3.4$ & $1.0 \pm 3.4$ & $1.1 \pm 3.5$ & $1.0 \pm 3.4$ \\
\hline & Oligochaeta & Oligochaeta s/i & $5.0 \pm 9.2$ & $18.4 \pm 43.1$ & 0 & $32.0 \pm 49.4$ \\
\hline \multirow[t]{24}{*}{ Arthropoda } & Amphipoda & Hyalellidae & $1 \pm 3.4$ & $1.0 \pm 3.4$ & 0 & 0 \\
\hline & Coleoptera & Elmidae & 0 & $2.9 \pm 7.2$ & $2.1 \pm 4.7$ & $4.8 \pm 6.0$ \\
\hline & Collembola & Collembola s/i & $1.9 \pm 4.5$ & 0 & $1.1 \pm 3.5$ & 0 \\
\hline & Decapoda & Aeglidae & 0 & $1.0 \pm 3.4$ & 0 & 0 \\
\hline & Diptera & Chironomidae & $32.0 \pm 31.4$ & $86.2 \pm 111.0$ & $26.4 \pm 23.3$ & $16.5 \pm 16.0$ \\
\hline & & $\begin{array}{c}\text { Chiron. } \\
\text { Tanypodinae }\end{array}$ & $26.2 \pm 24.4$ & $22.3 \pm 26.9$ & $22.2 \pm 34.0$ & $10.7 \pm 18.2$ \\
\hline & & $\begin{array}{c}\text { Chiron. } \\
\text { Orthocladiinae }\end{array}$ & $25.2 \pm 40.8$ & $20.4 \pm 38.1$ & $7.4 \pm 9.4$ & $9.7 \pm 12.0$ \\
\hline & & $\begin{array}{c}\text { Chiron. } \\
\text { Chironominae }\end{array}$ & 0 & $11.0 \pm 36.9$ & $7.4 \pm 15.0$ & $4.8 \pm 9.2$ \\
\hline & & $\begin{array}{l}\text { Chiron. Chiron. } \\
\text { Tanytarsini }\end{array}$ & $27.1 \pm 52.1$ & $41.7 \pm 78.5$ & $23.3 \pm 26.5$ & $11.6 \pm 16.4$ \\
\hline & & $\begin{array}{l}\text { Chiron. Chiron. } \\
\text { Chironomini }\end{array}$ & 0 & 0 & 0 & $1.9 \pm 4.5$ \\
\hline & & Ceratopogonidae & 0 & 0 & $1.1 \pm 3.5$ & 0 \\
\hline & Ephemeropotera & Ephemeroptera s/i & $5.8 \pm 20.1$ & $3.9 \pm 10.3$ & 0 & $1.0 \pm 3.4$ \\
\hline & & Baetidae & $20.4 \pm 33.3$ & $9.7 \pm 14.0$ & $2.1 \pm 4.7$ & $2.9 \pm 5.3$ \\
\hline & & Caenidae & $19.4 \pm 19.3$ & $17.4 \pm 21.4$ & $1.1 \pm 3.5$ & $1.0 \pm 3.4$ \\
\hline & & Leptohyphidae & $56.2 \pm 31.7$ & $70.7 \pm 63.0$ & $38.1 \pm 19.5$ & $43.0 \pm 29.5$ \\
\hline & & Leptophlebiidae & $26.2 \pm 14.1$ & $17.4 \pm 15.3$ & $7.4 \pm 9.4$ & $3.9 \pm 10.3$ \\
\hline & Hemiptera & Hemiptera s/i & $1.0 \pm 3.4$ & 0 & 0 & 0 \\
\hline & & Corixidae & $1.0 \pm 3.4$ & 0 & $1.1 \pm 3.5$ & 0 \\
\hline & Odonata & Zygoptera & $1.9 \pm 4.5$ & $1.0 \pm 3.4$ & $5.3 \pm 8.0$ & $5.8 \pm 9.3$ \\
\hline & Oribatida & Oribatida s/i & 0 & 0 & $1.1 \pm 3.5$ & 0 \\
\hline & Trichoptera & Trichoptera s/i & $3.9 \pm 7.6$ & $2.9 \pm 7.2$ & $10.6 \pm 11.0$ & $12.6 \pm 15.3$ \\
\hline & & Polycentropodidae & $1.9 \pm 4.5$ & $3.9 \pm 7.6$ & $8.5 \pm 14.8$ & $8.7 \pm 11.2$ \\
\hline & & Philopotamidae & 0 & 0 & 0 & $2.9 \pm 7.2$ \\
\hline & Trombidiformes & Hydrachnidae & $3.9 \pm 5.7$ & $1.0 \pm 3.4$ & 0 & 0 \\
\hline \multirow[t]{10}{*}{ Mollusca } & Bivalvia & Limnoperna fortunei & $228.4 \pm 163.1$ & $307.8 \pm 156.4$ & $6943.3 \pm 3869.0$ & $6943.3 \pm 3869.0$ \\
\hline & Gastropoda & $\begin{array}{l}\text { Pomacea } \\
\text { megastoma }\end{array}$ & 0 & 0 & 0 & $1.0 \pm 3.4$ \\
\hline & & Chilinidae & $10.7 \pm 11.6$ & $21.3 \pm 26.3$ & $5.3 \pm 10.9$ & $2.9 \pm 5.3$ \\
\hline & & Heleobia spp. & $14.5 \pm 39.7$ & $29.1 \pm 31.9$ & $29.6 \pm 35.7$ & $35.9 \pm 36.3$ \\
\hline & & Planorbidae & 0 & 0 & $1.1 \pm 3.5$ & $1.0 \pm 3.4$ \\
\hline & & Potamolithus spp. & $929.3 \pm 611.5$ & $\begin{array}{l}1191.9 \pm \\
796.5\end{array}$ & $1204.0 \pm 763.0$ & $556.2 \pm 302.2$ \\
\hline & Sphaeriida & Eupera sp. & 0 & 0 & $1.1 \pm 3.5$ & 0 \\
\hline & Veneroida & Corbiculidae & 0 & 0 & $1.0 \pm 3.4$ & 0 \\
\hline & & Densidad total \pm ds & $1209 \pm 571$ & $1561 \pm 718$ & $1432 \pm 729$ & $791 \pm 355$ \\
\hline & & Riqueza total (S) & 22 & 22 & 24 & 23 \\
\hline
\end{tabular}

A partir de los modelos ajustados, se encontró que existe relación lineal negativa significativa entre las abundancias de los grupos de invertebrados bentónicos nativos más frecuentes y las abundancias de $L$. fortunei. Específicamente, se encontró que las abundancias de gasterópodos, dípteros y efemerópteros se reducen significativamente a medida que las abundancias de mejillones aumentan $(p=0,006 ; 0,013 ; 0,0001$, respectivamente) (Tabla 2; Figura 
$4 a, b$ y c). Contrariamente, las abundancias de tricópteros aumentan conforme se incrementa la abundancia de mejillón dorado $(p=0,001)$ (Tabla 2; Figura 4d).

Tabla 2. Modelos lineales generalizados de efectos mixtos (GLMM, por sus siglas en inglés) ajustados para la abundancia de cada grupo de macroinvertebrados nativos como variable de respuesta, utilizando la abundancia de mejillón como variable explicativa de efecto fijo y la jaula como variable de efecto aleatorio. Se detalla el modelo seleccionado, su significancia ( $p$-valor) y F-valor, y el porcentaje de varianza explicada por el mismo.

\begin{tabular}{|ccccc|}
\hline $\begin{array}{c}\text { Variable } \\
\text { de respuesta }\end{array}$ & $\begin{array}{c}\text { Modelo } \\
\text { final }\end{array}$ & F & p & $\begin{array}{c}\text { \% de varianza } \\
\text { explicada }\end{array}$ \\
\hline Gastropoda & GLMM binom. neg. & 8,58 & 0,006 & $26 \%$ \\
\hline Diptera & GLMM binom. neg. & 6,27 & 0,013 & $19 \%$ \\
\hline Ephemeroptera & GLMM binom. neg. & 13,17 & 0,0001 & $26 \%$ \\
\hline Trichoptera & GLMM poisson & 10,81 & 0,001 & $14 \%$ \\
\hline
\end{tabular}




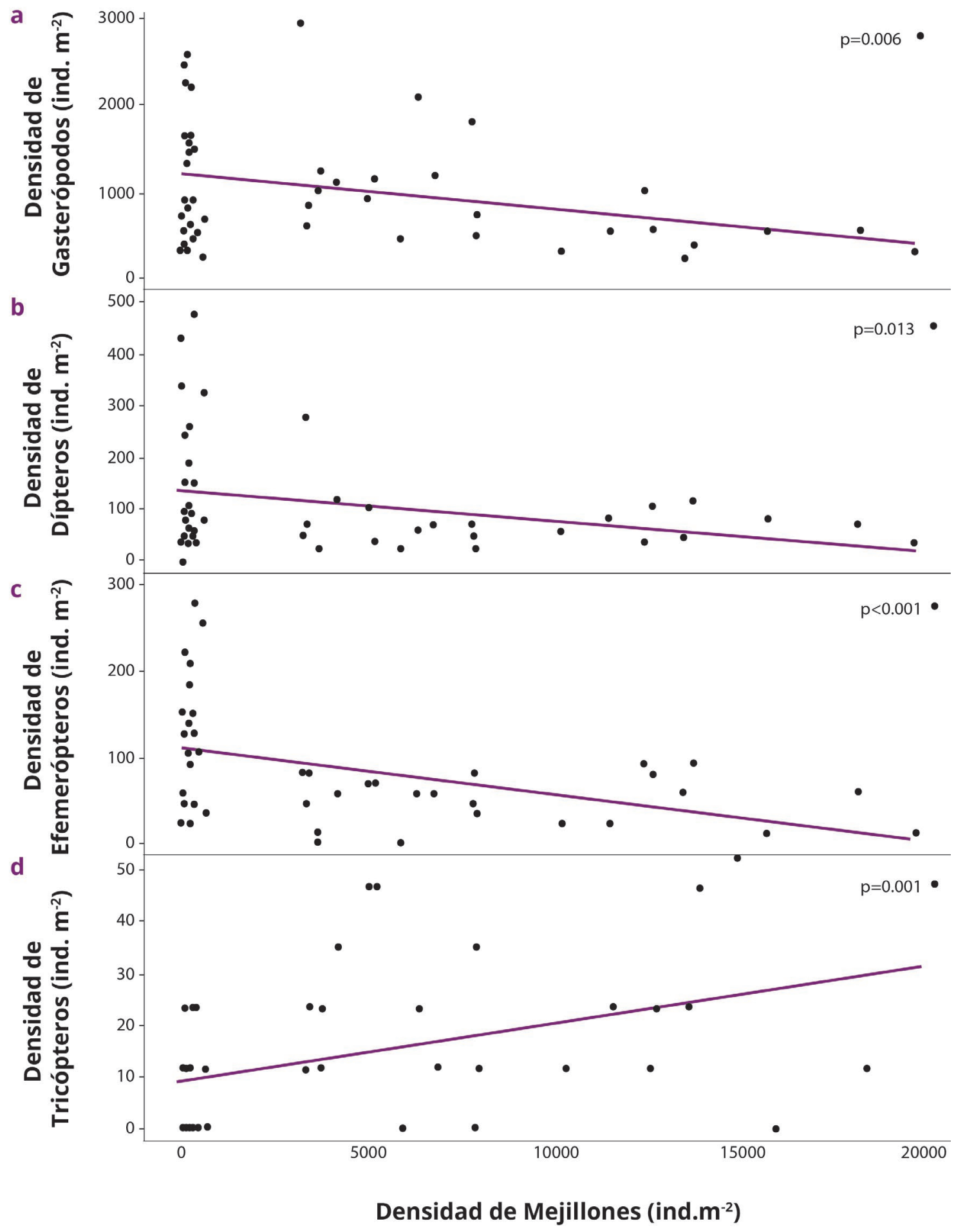

Figura 4. Densidad de cada grupo de macroinvertebrados bentónicos frecuentes del bajo Río Uruguay en función de la abundancia de mejillones. Se indica la significancia del modelo (y de las relaciones encontradas) por medio del $p$-valor. 


\section{DISCUSIÓN}

A pesar de que las implicancias ecosistémicas de la invasión del mejillón dorado han sido estudiadas en sistemas dulceacuícolas de Sudamérica, como el Río Paraná o el Río de la Plata (Mansur, et al., 1999; 2003; Sylvester, et al., 2007; Boltovskoy y Correa, 2015; Linares, et al., 2017; Duchini, et al., 2018), la información existente sobre las consecuencias directas e indirectas de este invasor sobre la fauna bentónica nativa al momento es escasa y poco difundida. En este trabajo, se sintetiza la información encontrada sobre los principales efectos que tiene la invasión de este mejillón sobre grupos particulares de macroinvertebrados bentónicos nativos del Río Uruguay, el sistema hídrico con mayor caudal de nuestro país.

Mediante la búsqueda bibliográfica y los registros fotográficos reportados, se encontró específicamente que los mejillones interactúan con varios grupos de moluscos autóctonos (incluyendo gasterópodos y bivalvos). A través del biofouling provocan problemas en la locomoción, y un aumento del riesgo de depredación, ya que se obstruyen las estructuras de protección del organismo (por ejemplo, opérculos, valvas) (Eugui, et al., 2012). Estos registros son consistentes con otros reportes previos para otros sistemas. Por ejemplo, Mansur y otros (1999) encontraron ejemplares de mejillones fijados al caparazón de Corbicula spp. y de Diplodon koseritzi próximos a las aberturas de inhalación y exhalación, pudiendo provocar el sofocamiento de estos organismos. Además, Mansur y otros (2003) también reportaron interacciones entre el mejillón y otros moluscos: por ejemplo, se ha documentado la bioincrustración de este invasor sobre el bivalvo L. blainvilliana, y en gasterópodos, como Pomacea canaliculata. Estos reportes también son consistentes con lo indicado por Scarabino (2004), quien establece por medio de una revisión bibliográfica los registros del biofouling generado por Limnoperna sobre la malacofauna de varios sistemas de agua dulce sudamericanos. En este sentido, Soutullo y otros (2013) han destacado la implicancia de este invasor como uno de los principales factores de riesgo para los grupos de moluscos prioritarios para la conservación en Uruguay.

Por otro lado, las interacciones observadas con los poríferos nativos parecen ser negativas para los mejillones, ya que tanto el registro fotográfico como las observaciones de campo muestran especímenes del mejillón dorado que quedan completamente cubiertos por la esponja, imposibilitando su apertura de valvas. Más aún, en el interior de las colonias de poríferos se observan valvas cerradas de mejillones muertos (Iván González-Bergonzoni, obs. pers.). Si bien las interacciones con diferentes taxa que componen la fauna bentónica se han inferido con base en extensas revisiones bibliográficas (Sylvester y Sardiña, 2015), en ellas no se reporta la posible interacción entre el mejillón dorado y esponjas de agua dulce. Este registro de interacción entre el mejillón dorado y especies de 
Porífera del Río Uruguay, en conjunto con observaciones similares registradas recientemente en afluentes de Brasil (Fortunato, 2021), resulta sumamente novedoso para la región. Durante el comienzo de la invasión del mejillón dorado en el Río Paraná, un estudio sobre esponjas de agua dulce de Argentina discutió el potencial efecto negativo del invasor $L$. fortunei, dado que posiblemente ambos compitan por el alimento y por el espacio (Ezcurra de Drago, 2004). Sin embargo, si bien esto es cierto, las observaciones aquí realizadas sugieren que en una competencia por el espacio las esponjas son capaces de desplazar (cubriendo totalmente) a los mejillones.

Las aproximaciones experimentales realizadas en el bajo Río Uruguay (Silva, et al., 2020), en conjunto con los resultados aquí descritos, destacan que efectivamente la colonización masiva de mejillones ocasiona consecuencias para la fauna de macroinvertebrados nativos, consideradas negativas para algunos grupos (por ejemplo, gasterópodos y dípteros) y positivas para otros (por ejemplo, tricópteros). Específicamente, se encontró que las abundancias de varios de los grupos de macroinvertebrados bentónicos se correlacionan con las abundancias de mejillones. Particularmente, se destaca que los gasterópodos, dípteros y efemerópteros pueden verse seriamente afectados ante esta invasión, disminuyendo significativamente sus abundancias conforme aumenta la abundancia del mejillón en los sustratos. Las causas por las cuales estos invertebrados podrían verse tan afectados ante dicha invasión aún no han sido estudiadas en detalle. Sin embargo, podría sugerirse que además de las bioincrustaciones realizadas sobre grandes gasterópodos y bivalvos nativos (Darrigran, et al., 1998), la mayor acumulación de materia orgánica en el sustrato bentónico, producido por las heces y pseudoheces de miles de mejillones, implicaría un menor acceso de luz, generando una afectación en el crecimiento de las algas bentónicas y, por lo tanto, una disminución en el perifiton disponible en los sustratos adyacentes a las colonias de $L$. fortunei. El perifiton consiste en el alimento principal de muchos gasterópodos, efemerópteros y quironómidos (Cummins y Klug, 1979). Una menor disponibilidad de este recurso podría provocar un desplazamiento de estos taxa.

Por otra parte, se encontró que la abundancia de tricópteros aumentó ante la presencia de mejillones en los sustratos. Esto coincide con la mayoría de las investigaciones previas (Boltovskoy, et al., 2009; Karatayev, et al., 2010; Burlakova, et al., 2012; Duchini, et al., 2018) que señalan a la capacidad de L. fortunei como facilitadora de varios grupos de invertebrados nativos en el río Paraná y Río de la Plata. Estos estudios destacan que algunos grupos con hábitos alimenticios de tipo sedimentívoro (por ejemplo, Oligochaeta y Nematoda) se ven favorecidos por la presencia del mejillón dorado, aumentando significativamente sus abundancias luego de la invasión. Esta facilitación podría estar asociada a una mayor disponibilidad de alimento por enriquecimiento de los sedimentos con 
materia orgánica (Sardiña, et al., 2008; Boltovskoy y Correa, 2015) (Figura 5). En dichos trabajos también se destaca el aumento de organismos predadores, como los hirudíneos, ante la presencia de mejillones, lo cual podría estar dado por una mayor complejidad estructural que brindaría mayores oportunidades de "caza".

Si bien en este estudio no se determinó en detalle la taxonomía, desconociendo los hábitos funcionales de los tricópteros colectados, podría sugerirse que los mismos estarían siendo beneficiados por el aporte alimenticio y estructural que brindan las colonias de mejillones. Sin embargo, se vuelve necesaria una identificación más detallada para poder obtener conclusiones más certeras al respecto.

De acuerdo con la información recopilada en la revisión bibliográfica y en estudios propios, se sugiere que la invasión del mejillón dorado en grandes ríos, y específicamente en el Río Uruguay, implica diversas consecuencias para las comunidades bentónicas, dadas por una compleja interacción de diferentes mecanismos. En este sentido, se generan efectos positivos para algunos macroinvertebrados nativos, y negativos para otros grupos (Figura 5). Por ejemplo, algunos de los efectos positivos hallados estriban en la alteración del hábitat próximo a las colonias, que se vuelve favorable para quienes puedan refugiarse $y / o$ alimentarse en los espacios intersticiales, altamente ricos en materia orgánica. Por otro lado, la modificación indirecta de los parámetros fisicoquímicos del agua (afectando la transparencia y el balance de nutrientes en agua; Cataldo, et al., 2012b) podría favorecer a productores primarios en zonas más alejadas de las colonias y a sus consumidores (como se discute en Boltovskoy, et al., 2015). En cuanto a los efectos negativos, quizás los más evidentes sean los efectos directos causados por el biofouling sobre moluscos y quizás también sobre crustáceos nativos, con consecuencias claras para su movilidad y desarrollo. Sin embargo, los efectos de alteración de hábitat que ocurre en la zona de las colonias y sus adyacencias parecen ser un mecanismo relevante por el cual $L$. fortunei afecta negativamente a los gasterópodos, dípteros y efemerópteros (Figura 5).

No obstante, debido a que estos análisis fueron realizados considerando dos muestreos diferentes, también es importante considerar otros factores que puedan tener implicancias en la variación de las abundancias de invertebrados, por ejemplo, el ciclo natural de cada grupo. En este sentido, muchos de estos organismos pueden potencialmente emerger en verano, provocando una reducción de las larvas acuáticas en los meses de mayor temperatura del agua (coincidiendo con los días en que se llevó a cabo el experimento). Por tanto, el patrón de abundancias de la fauna bentónica nativa no se puede atribuir estrictamente a la presencia de mejillones, aunque los registros puntuales, investigaciones previas y los resultados del análisis aquí expuesto sugieren que la 
presencia de este invasor en los cursos de la Cuenca del Plata tendría especial relevancia en la dinámica de las comunidades de macroinvertebrados bentónicos.

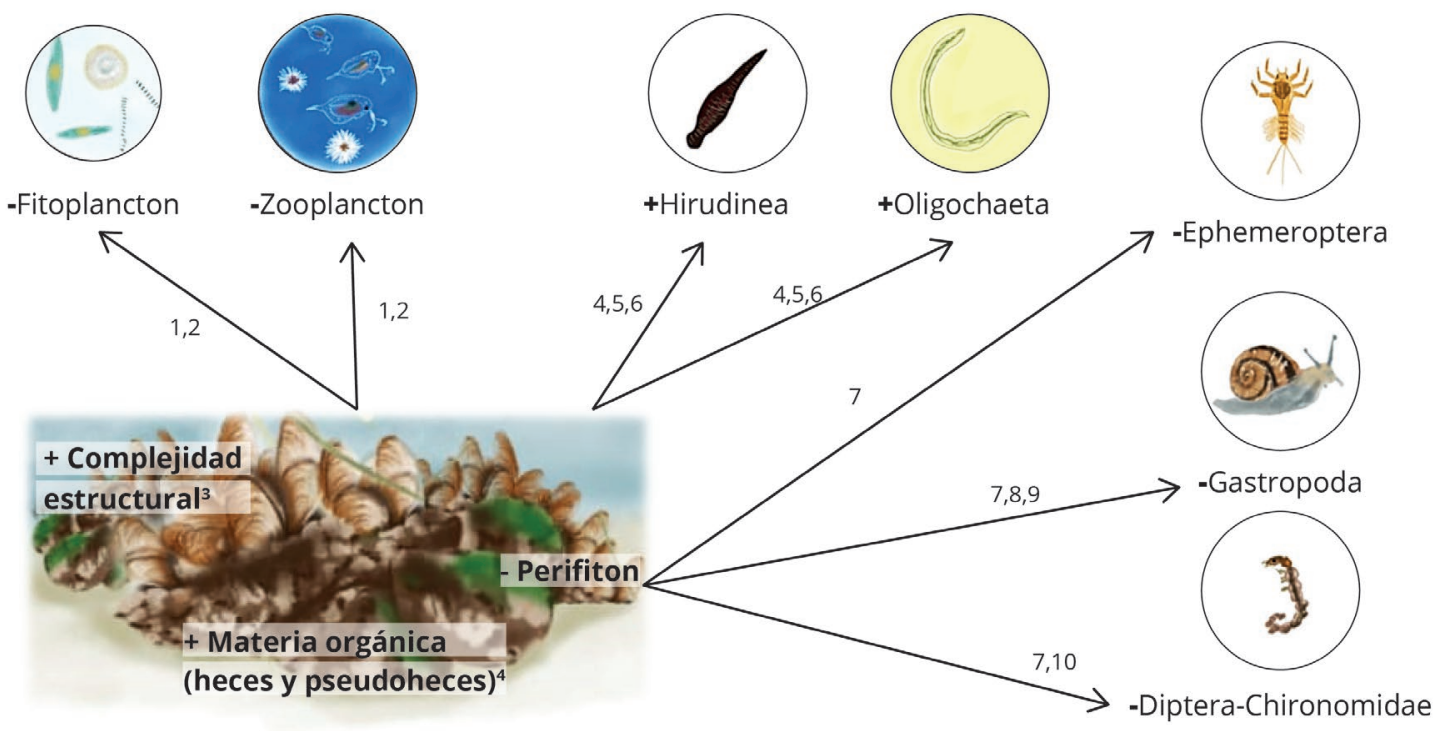

\section{Limnoperna fortunei}

Figura 5. Implicancias de la invasión del mejillón dorado sobre el ecosistema y principales consecuencias positivas (+) y negativas (-) sobre los invertebrados bentónicos nativos. Se indica con un número cada referencia: (1) Cataldo, et al., 2012a; (2) Sylvester, et al., 2005; (3) Sardiña, et al., 2008; (4) Boltovskoy y Correa, 2015; (5) Sylvester, et al., 2007; (6) Duchini, et al., 2018; (7) Silva, et al., 2020; (8) Mansur, et al., 2003; (9) Darrigran, et al., 1998; (10) Linares, et al., 2017.

\section{CONCLUSIÓN}

Si bien las consecuencias económicas de la invasión del mejillón dorado son frecuentemente analizadas y cuantificadas, poco se conoce sobre el impacto ecológico del mejillón dorado en los sistemas dulceacuícolas platenses. Por ello, con este trabajo se intenta contribuir a un mejor entendimiento de dichos impactos ecológicos. En este sentido, se considera necesaria la realización de estudios que profundicen en la ecología de la invasión de este bivalvo, lo cual permitirá proponer posibles medidas de mitigación de sus principales efectos, en políticas de gestión y manejo de esta plaga.

\section{AGRADECIMIENTOS}

Agradecemos a Maite Burwood, Tatiana Vernasa, Samanta Stebniki, Clementina Calvo y Nicolás Vidal por ayudar en las campañas de muestreo. También agradecemos a los pescadores artesanales Elbio y Julio Russo, que amablemente ayudaron a cuidar los módulos experimentales desplegados en el río, y a Gonzalo Hernández-Fripp, por las obras de arte utilizadas en las figuras del manuscrito. 
Este proyecto de investigación fue financiado por la Agencia Nacional de Innovación e Investigación (ANII), proyecto ANII FCE2 2016-1-126780 y la beca ANII PD_NAC_2015_1_108121. Silva recibió apoyo financiero del programa de becas de la Comisión Académica de Posgrado (CAP) y del PEDECIBA. GonzálezBergonzoni, Naya, D'Anatro, Clavijo, Brugnoli, Tesitore y Teixeira de Mello recibieron apoyo financiero de ANII y del PEDECIBA.

\section{REFERENCIAS}

Boltovskoy, D. y Correa, N., 2015. Ecosystem impacts of the invasive bivalve Limnoperna fortunei (golden mussel) in South America. En: Hydrobiologia, 746, pp.81-95. DOI: https://doi.org/10.1007/s10750-014-1882-9

Boltovskoy, D., Karatayev, A., Burlakova, L., Cataldo, D., Karatayev, V., Sylvester, F. y Marinelarena, A., 2009. Significant ecosystem-wide effects of the swiftly spreading invasive freshwater bivalve Limnoperna fortunei. En: Hydrobiologia, 636(1), pp.271-284. DOI: https://doi.org/10.1007/s10750$\underline{\text { 009-9956-9 }}$

Boltovskoy, D., Xu, M. y Nakano, D., 2015. Impacts of Limnoperna fortunei on man-made structures and control strategies: general overview. En: Boltovskoy, D., ed. Limnoperna fortunei: the ecology, distribution and control of a swiftly spreading invasive fouling mussel. Cham: Springer. pp. 375-393. DOI: https://doi.org/10.1007/978-3-319-13494-9 21

Brugnoli, E., Clemente, J., Boccardi, L., Borthagaray, A. y Scarabino, F., 2005. Golden mussel Limnoperna fortunei (Bivalvia, Mytilidae) distribution in the principal hydrographic basin of Uruguay: Update and prediction. En: Anais da Academia Brasileira de Ciências, 77(2), pp.235-244. DOI: https://doi.org/10.1590/S0001-37652005000200004

Brugnoli, E., Clemente, J., Riestra, G., Boccardi, L. y Borthagaray, A., 2006. Especies acuáticas exóticas en Uruguay: situación, problemática y gestión. En: Menafra, R., Rodríguez, L., Scarabino, F. y Conde, D., eds. Bases para la conservación y manejo de la costa uruguaya. Montevideo: Vida Silvestre Uruguay. pp.351-362.

Brugnoli, E., Dabezies, M.J., Clemente, J. y Muniz, P., 2011. Limnoperna fortunei (Dunker 1857) en el sistema de embalses del Río Negro, Uruguay. En: Oecologia Australis, 15, pp.576-592. DOI: 10.4257/oeco.2011.1503.10 
Burlakova, L.E., Karatayev, A.Y. y Karatayev, V.A., 2012. Invasive mussels induce community changes by increasing habitat complexity. En: Hydrobiologia, 685, pp.121-134. DOI: https://doi.org/10.1007/s10750-011-0791-4

Cataldo, D.H., Boltovskoy, D. y Pose, M., 2003. Toxicity of chlorine and three non-oxidising molluscicides to the pest mussel Limnoperna fortunei. En: Journal of the American Waterworks Association, 95, pp.66-78. DOI: https://doi.org/10.1002/j.1551-8833.2003.tb10270.x

Cataldo, D., O’Farrell, I., Paolucci, E., Sylvester, F. y Boltovskoy, D., 2012a. Impact of the invasive golden mussel (Limnoperna fortunei) on phytoplankton and nutrient cycling in South America. En: Aquatic Invasions, 7, pp.91-100. DOI: 10.3391/ai.2011.ICAIS

Cataldo, D., Vinocur, A., Inés, O., Paolucci, E., Leites, V. y Boltovskoy, D., 2012b. The introduced bivalve Limnoperna fortunei boosts Microcystis growth in Salto Grande reservoir (Argentina): evidence from mesocosm experiments. En: Hydrobiologia, 680(1), pp.25-38. DOI: https://doi.org/10.1007/s10750-011-0897-8

Clemente, J., Iglesias, C., Kröger, A. y José, J., 2015. First record of the golden mussel Limnoperna fortunei Dunker, 1857 (Bivalvia: Mytilidae) in a lentic system in Uruguay. En: Pan-American Journal of Aquatic Sciences, 10(1), pp.89-93.

Costa, C., Ide, S. y Simonka, C.E., 2006. Insetos imaturos: metamorfose e identificação. Ribeirão Preto: Holos Editora.

Crooks, J.A., 2002. Characterizing ecosystem-level consequences of biological invasions: the role of ecosystem engineers. En: Oikos, 97, pp.153-166.

Cummins, K.W. y Klug, M.J., 1979. Feeding ecology of stream invertebrates. En: Annual Review of Ecology and Systematics, 10(1), pp.147-172.

Darrigran, G., 2002. Potential impact of filter-feeding invaders on temperate inland freshwater environments. En: Biological Invasions, 4(1-2), pp.145156. DOI: https://doi.org/10.1023/A:1020521811416

Darrigran, G., Martin, S.M., Gullo, B. y Armendariz, L., 1998. Macroinvertebrates associated with Limnoperna fortunei (Dunker, 1857) (Bivalvia, Mytilidae) in Río de la Plata, Argentina. En: Hydrobiologia, 367(1-3), pp.223-230. 
de Melo Rosa, D., da Costa Gaspar, M.R., Silva, F.A. y Pompeu, P.S., 2019. Impacts of predation by piapara Megaleporinus obtusidens (Valenciennes, 1837) on the population densities of the invasive golden mussel Limnoperna fortunei (Dunker, 1857). En: Biological control, 129, pp.158-163. DOI: https://doi.org/10.1016/j.biocontrol.2018.10.012

Delignette-Muller, M.L. y Dutang, C., 2015. Fitdistrplus: an R package for fitting distributions. En: Journal of Statistical Software, 64, pp.1-34. DOI: 10.18637/jss.v064.i04

Domínguez, E. y Fernández, H.R., eds., 2009. Macroinvertebrados bentónicos sudamericanos. Tucumán: Fundación Miguel Lillo.

Domínguez, E., Molineri, C., Pescador, M.L., Hubbard, M.D. y Nieto, C., 2006. Ephemeroptera de América del Sur. En: Adis, J., Arais, J.R., Rueda-Delgado, G. y Wantzen, K.M., eds. Biodiversidad acuática en América Latina. Vol. II. Sofía: Pensoft.

Duchini, D., Boltovskoy, D. y Sylvester, F., 2018. The invasive freshwater bivalve Limnoperna fortunei in South America: multiannual changes in its predation and effects on associated benthic invertebrates. En: Hydrobiologia, 817, pp.431-446. DOI: https://doi.org/10.1007/s10750-018-3561-8

Eugui, F., Clavijo, C., Röhrdanz, A., Calvo, C., Gibson, K., Plantz, A., Scarabino, F., Carranza, A., Meerhoff, M. y Burks, R., 2012. Macrofouling del mejillón dorado Limnoperna fortunei (Bivalvia) sobre Ampullariidae (Gastropoda) en aguas continentales del Uruguay. En: Sociedad Zoológica del Uruguay. Libro de resúmenes del II Congreso Uruguayo de Zoología. Montevideo: Sociedad Zoológica del Uruguay. pp.69.

Ezcurra de Drago, I., 2004. Biodiversidad de Porifera en el Litoral Argentino. Grado de competencia con el bivalvo invasor Limnoperna fortunei (Dunker, 1857) (Bivalvia, Mytilidae). En: Miscelánea, 12, pp.195-204. ISSN 1514-4836 - ISSN On-Line 1668-3242

Ezcurra de Drago, I., Montalto, I. y Oliveros, O., 2006. Desarrollo y ecología larval de Limnoperna fortunei. En: Darrigran, G. y Damborenea, C., eds. Bioinvasión del mejillón dorado en el continente americano. La Plata: Universidad Nacional de la Plata. 
Fortunato, H., 2021. Impacto ecológico do mexilhão dourado sobre a comunidade bentónica. En: 3er Worshop: Mexilhão dourado: uma ameaça ao ambiente e à sociedade. Projeto Mexilhão dourado um compromisso com o Brasil. Brasil (19 de marzo de 2021). [s.l.]. [s.n.]

Karatayev, A.Y., Burlakova, L.E., Karatayev, V.A. y Boltovskoy, D., 2010. Limnoperna fortunei versus Dreissena polymorpha: population densities and benthic community impacts of two invasive freshwater bivalves. En: Journal of Shellfish Research, 29, pp.975-984. DOI: https://doi.org/10.2983/035.029.0432

Linares, M.S., Callisto, M. y Marques, J.C., 2017. Invasive bivalves increase benthic communities complexity in neotropical reservoirs. En: Ecological Indicators, 75, pp.279-285. DOI: https://doi.org/10.1016/j.ecolind.2016.12.046

Logan, M., 2010. Biostatistical design and analysis using $R$ : a practical guide. Chichester: Wiley-Blackwell.

Lopes, M., Vieira, J.P. y Burns, M.D., 2009. Biofouling of the golden mussel Limnoperna fortunei (Dunker, 1857) over the Anomura crab Aegla platensis Schmitt, 1942. En: Pan-American Journal of Aquatic Sciences, 4(2), pp.222225.

Mansur, M.C.D., dos Santos, C.P., Darrigran, G., Heydrich, I., Callil, C.T. y Cardoso, F.R., 2003. Primeiros dados quali-quantitativos do mexilhão-dourado, Limnoperna fortunei (Dunker), no Delta do Jacuí, no Lago Guaíba e na Laguna dos Patos, Rio Grande do Sul, Brasil e alguns aspectos de sua invasão no novo ambiente. En: Revista Brasileira de Zoologia, 20(1), pp.7584. DOI: $\underline{\text { https://doi.org/10.1590/S0101-81752003000100009 }}$

Mansur, M.C.D., Richinitti, L.M.Z. y dos Santos, C.P., 1999. Limnoperna fortunei (Dunker, 1857) molusco bivalve invasor na bacia do Guaíba, Rio Grande do Sul, Brasil. En: Biociências, 7(2), pp.147-149.

Pastorino, G., Darrigran, G.A., Martin, S.M. y Lunaschi, L., 1993. Limnoperna fortunei (Dunker, 1857) (Mytilidae), nuevo bivalvo invasor en aguas del Río de la Plata. En: Neotropica, 39, pp.34.

Pérez, M.C., 2012. Las incrustaciones biológicas (biofouling) y metodología para su control. La Plata: Universidad Nacional de La Plata. (Disertación Doctoral). 
Rojas Molina, F.R. y Williner, V., 2013. First record of the non-indigenous mussel Limnoperna fortunei (Bivalvia, Mytilidae) as an epibiont of the crab Trichodactylus borellianus (Decapoda, Trichodactylidae). En: Crustaceana, 86(6), pp.682-692. DOI: http://dx.doi.org/10.1163/15685403-00003183

Sardiña, P., Cataldo, D. y Boltovskoy, D., 2008. The effects of the invasive mussel, Limnoperna fortunei, on associated fauna in South American freshwaters: importance of physical structure and food supply. En: Fundamental and Applied Limnology/Archiv fur Hydrobiologie, 173, pp.135-144. DOI: 10.1127/1863-9135/2008/0173-0135

Scarabino, F., 2004. Conservación de la malacofauna uruguaya. En: Comunicaciones de la Sociedad Malacológica del Uruguay, 8(83), pp.267273.

Scarabino, F. y Verde, M., 1995. Limnoperna fortunei (Dunker 1857) en la costa uruguaya del Río de la Plata (Bivalvia; Mytilidae). En: Comunicaciones de la Sociedad Malacológica del Uruguay, 7, pp.374-375.

Silva, I., Naya, D., Teixeira de Mello, F., D'Anatro, A., Tesitore, G.; Clavijo, C. y González-Bergonzoni, I., 2020. Fish vs. aliens: predatory fish regulate populations of Limnoperna fortunei mitigating impacts on native macroinvertebrates communities. En: Hydrobiología, pp.1-21. DOI: https://doi.org/10.1007/s10750-020-04421-9

Soutullo, A., Clavijo, C. y Martínez-Lanfranco, J.A., eds., 2013. Especies prioritarias para la conservación en Uruguay. Vertebrados, moluscos continentales y plantas vasculares. Montevideo: SNAP, DINAMA, MVOTMA, MEC.

Sylvester, F., Boltovskoy, D. y Cataldo, D., 2007. The invasive bivalve Limnoperna fortunei enhances benthic invertebrate densities in South American floodplain rivers. En: Hydrobiologia, 589(1), pp.15-27. DOI: https://doi.org/10.1007/s10750-007-0708-4

Sylvester, F., Dorado, J., Boltovskoy, D., Juarez, A. y Cataldo, D., 2005. Filtration rates of the invasive pest bivalve Limnoperna fortunei as a function of size and temperature. En: Hydrobiologia, 534, pp.71-80. DOI: https://doi.org/10.1007/s10750-004-1322-3 
Sylvester, F. y Sardiña, P., 2015. Relationships of Limnoperna fortunei with benthic animals. En: Boltovskoy, D., ed. Limnoperna Fortunei. The ecology, distribution and control of a swiftly spreading invasive fouling mussel. Cham: Springer. (Invading Nature - Springer Series in Invasion Ecology, vol. 10). pp.191-210. DOI: https://doi.org/10.1007/978-3-319-13494-9 11

Welker, M. y Walz, N., 1998. Can mussels control the plankton in rivers? a planktological approach applying a Lagrangian sampling strategy. En: Limnology and Oceanography, 43(5), pp.753-762. DOI: https://doi.org/10.4319/lo.1998.43.5.0753 